\title{
P-glycoprotein alters blood-brain barrier penetration of antiepileptic drugs in rats with medically intractable epilepsy
}

This article was published in the following Dove Press journal:

Drug Design, Development and Therapy

2 December 2013

Number of times this article has been viewed

\section{Aimei Ma ${ }^{1, *}$ \\ Cuicui Wang ${ }^{2,3, *}$ \\ Yinghui Chen ${ }^{2,3}$ \\ Weien Yuan ${ }^{4}$}

'Department of Neurology, The People's Hospital of Shanxi Province, Taiyuan, ${ }^{2}$ Department of Neurology, Jinshan Hospital, Fudan University, ${ }^{3}$ Department of Neurology, Shanghai Medical College, Shanghai, ${ }^{4}$ School of Pharmacy, Shanghai JiaoTong University, Shanghai, People's Republic of China

*These authors contributed equally to this work

Correspondence: Yinghui Chen Department of Neurology, Jinshan Hospital, Fudan University, Shanghai 20I508, People's Republic of China Email yinghuichen@fudan.edu.cn

Weien Yuan

School of Pharmacy, Shanghai JiaoTong University, 800 Dongchuan Road, Shanghai 200240, People's Republic of China

Email yuanweien@I26.com
Abstract: P-glycoprotein is one of the earliest known multidrug transporters and plays an important role in resistance to chemotherapeutic drugs. In this study, we detected levels of P-glycoprotein and its mRNA expression in a rat brain model of medically intractable epilepsy established by amygdala kindling and drug selection. We investigated whether inhibition of P-glycoprotein affects the concentration of antiepileptic drugs in cortical extracellular fluid. We found that levels of P-glycoprotein and its mRNA expression were upregulated in epileptic cerebral tissue compared with cerebral tissue from normal rats. The concentrations of two antiepileptic drugs, carbamazepine and phenytoin, were very low in the cortical extracellular fluid of rats with medically intractable epilepsy, and were restored after blockade of P-glycoprotein by verapamil. These results show that increased P-glycoprotein levels alter the ability of carbamazepine and phenytoin to penetrate the blood-brain barrier and reduce the concentrations of these agents in extracellular cortical fluid. High P-glycoprotein levels may be involved in resistance to antiepileptic drugs in medically intractable epilepsy.

Keywords: P-glycoprotein, medically intractable epilepsy, antiepileptic drugs, amygdala kindling, verapamil

\section{Introduction}

Although a number of new antiepileptic drugs have been launched over the past two decades, drug resistance remains a major problem. About $30 \%$ of patients are refractory to treatment with more than one antiepileptic drug. This so-called medically intractable epilepsy is often associated with a poor prognosis, ie, increased morbidity and mortality in patients. ${ }^{1-3}$ Therefore, it is important to investigate the mechanism of drug resistance in medically intractable epilepsy and develop new treatment strategies. Earlier research in the field of cancer has shown that the activity of P-glycoprotein and other ATP-binding cassette (ABC) transporters, such as multidrug resistance-associated proteins (MDRs), are directly related to drug resistance., ${ }^{4,5}$

MDR1-encoded P-glycoprotein is widely expressed in tissues with an excretory function, including the liver, kidneys, and cerebrum, as an energy-dependent efflux transporter, and is involved in barrier functioning, such as in the blood-brain barrier. ${ }^{5}$ In normal tissues, P-glycoprotein is thought to participate in the protection of cells from toxins or xenobiotics. In the brain, P-glycoprotein is predominantly located on the membrane of capillary endothelial cells which form the blood-brain barrier. Overexpression of P-glycoprotein on endothelial cells of the blood-brain barrier would limit penetration of antiepileptic drugs into the brain and reduce drug concentrations in 
cerebral tissue, suggesting that P-glycoprotein may be involved in mechanisms of drug resistance in patients with medically intractable epilepsy.

There is accumulating evidence showing that $\mathrm{P}$-glycoprotein is involved in resistance to antiepileptic drugs. Tishler et $\mathrm{al}^{6}$ were the first to report that P-glycoprotein is overexpressed in epileptogenic brain tissue from patients with pharmacoresistant partial epilepsy. More recently, P-glycoprotein was reported to be overexpressed in endothelial cells of the blood-brain barrier in a kainate model of temporal lobe epilepsy in rats. ${ }^{7}$ To confirm this phenomenon, expression of P-glycoprotein was studied by real-time reverse transcription polymerase chain reaction (RT-PCR) and Western blot technique in a rat model of medically intractable epilepsy created by amygdala kindling. ${ }^{8}$ Previous research has confirmed that the concentration of phenytoin, a classic antiepileptic drug, in extracellular cortical fluid is significantly increased by inhibition of P-glycoprotein, indicating that phenytoin is a substrate for P-glycoprotein. ${ }^{9}$ However, the opposite effect was observed for carbamazepine, another antiepileptic drug. ${ }^{10,11}$ To address this issue directly, we undertook a brain microdialysis experiment to study the impact of P-glycoprotein inhibition on penetration of antiepileptic drugs through the blood-brain barrier in rats with medically intractable epilepsy.

\section{Materials and methods}

\section{Materials}

Male Sprague Dawley rats (250-300 g) were obtained from the Shanghai Animal Center, Medical College of Fudan University, Shanghai, People's Republic of China, and maintained in the animal facility at $20^{\circ} \mathrm{C} \pm 2^{\circ} \mathrm{C}$ with a relative humidity of $60 \%$ and a 12-hour light and dark cycle for 5 days before the experiment. A total of 80 rats were used (four rats per cage). All rat experiments were carried out in accordance with the Guidelines for Animal Experiments of the Chinese Academy of Medical Sciences and with approval from the ethics committee for animal care at Jinshan Hospital.

Thirty-two rats were randomly assigned to either a control group $(n=16)$ or to a group with medically intractable epilepsy $(\mathrm{n}=16)$ for detection of P-glycoprotein expression, and a further 48 rats were randomly assigned to a phenytoin group $(n=24)$ or a carbamazepine group $(n=24)$ for a microdialysis experiment. The carbamazepine and phenytoin groups were further divided into three subgroups: a control group $(n=8)$ comprising normal rats that received intraperitoneal injection of antiepileptic drugs (carbamazepine $20 \mathrm{mg} / \mathrm{kg}$ or phenytoin $50 \mathrm{mg}$ / kg, Sigma-Aldrich, St Louis, MO, USA); an epilepsy group $(n=8)$ comprising kindled rats that received the same injections as the control group; and a verapamil group $(n=8)$ comprising kindled rats that received $50 \mu \mathrm{L}$ of verapamil $(20 \mathrm{mmol} / \mathrm{L}$, Sigma-Aldrich) at a rate of $2.5 \mu \mathrm{L}$ per minute from the inflow tube into the cortex 30 minutes before intraperitoneal antiepileptic drug injection.

\section{Establishment of a medically intractable epilepsy model}

The amygdala kindling model was generated using a well established protocol. ${ }^{12}$ Seizure severity was evaluated in accordance with the standards published by Racine. ${ }^{13}$ Rats with three consecutive stage $\mathrm{V}$ seizures were considered to be successfully kindled and were injected intraperitoneally with phenytoin to screen for resistance. Successful creation of a medically intractable epilepsy model was deemed to have occurred if there was a reduction in the after discharge threshold. The after discharge threshold was determined using an ascending series procedure whereby electrical kindling stimulation ( 2 seconds, $60 \mathrm{~Hz}$, biphasic square wave pulses) was started at $20 \mu \mathrm{A}$ and increased in steps of $20 \mu \mathrm{A}$ until an after discharge was triggered. The interval between stimulations was one minute. The after discharge threshold was defined as the minimum current intensity required to provoke a synchronous spike-and-wave pattern of at least 3 seconds in the electroencephalogram. Rats that did not respond to phenytoin were considered to have medically intractable epilepsy ${ }^{14}$ and electrode-implanted animals that did not display electrical kindling stimulation were used as the control group.

\section{RNA extraction and real-time RT-PCR}

Total RNA was extracted from rat brain tissue using TRIzol reagent (Invitrogen, New York, NY, USA). Total RNA (1 $\mu \mathrm{g})$ was reversely transcribed using a reverse transcription kit (MBI Fermentas, Burlington, OT, Canada), followed by polymerase chain reaction amplification. Primers for $M D R 1$ and $\beta$-actin were synthesized by Shanghai Saibaisheng Company (Shanghai, People's Republic of China). The primer sequences were: 5'-ACTCGGGAGCAGAAGTTTGA-3' (forward) and 5'-GGAGCCACTGGACATTGAGT-3' (reverse) for MDRl (600 bp); and 5'-AACCCTAAGGCCAACCGTGAAAAG-3' (forward) and 5'-TCATGAGGTAGTCTGTCAGGT-3' (reverse) for $\beta$-actin (241 bp). Thirty cycles of PCR were performed. The products of PCR were then separated on $1.5 \%$ agarose gel and analyzed using a gel imaging system (GeneGenius ${ }^{\circledR}$, Syngene Co, Ltd, Cambridge, UK).

\section{Western blotting}

Total protein was extracted from brain tissue using the following protocol. Equal amounts of protein samples were 
separated by sodium dodecyl sulfate polyacrylamide gel electrophoresis and transferred to polyvinylidene difluoride membranes. Each membrane was incubated using rabbit anti-rat primary antibody (1:500) and then horseradish peroxidase-conjugated goat anti-rabbit secondary antibody (1:2,000). Signals were determined using an ECL-Plus kit (GE Healthcare, Little Chalfont, Buckinghamshire, UK).

\section{Brain microdialysis}

Each rat was anesthetized by intraperitoneal injection of $1 \%$ pentobarbital sodium (Sigma-Aldrich) at $40 \mathrm{mg} / \mathrm{kg}$. A microdialysis probe was implanted according to stereotaxic coordinates. The head of the rat was then fixed in a stereotaxic apparatus (RWD Life Science Co, Ltd, Shenzhen, People's Republic of China) and a hole was drilled in the middle of the frontal cortex, $1 \mathrm{~mm}$ from the anterior fontanelle. A catheter was inserted via the hole into the cerebral cortex from the vertical line at a 30-degree angle to a depth of $3 \mathrm{~mm}$ and then fixed with dental cement. After connecting the microdialysis device, the catheter probe was inserted into the cerebral cortex of the rat. The catheter probe consists of a shaft with a semipermeable fiber membrane at its tip and was inserted into the cortex in its entirety. The microdialysis experiment was performed one week after surgical implantation of the probe.

The recovery efficiency of antiepileptic drugs was measured prior to each microdialysis. Briefly, the artificial cerebrospinal fluid rate with $20 \mu \mathrm{g} / \mathrm{mL}$ carbamazepine or phenytoin was injected using a microperfusion pump (KD100, KD Scientific Inc, Holliston, MA, USA) through the inflow tube, probe, and outflow tube at a constant flow rate $(2.5 \mu \mathrm{L}$ per minute), then balanced for 1 hour. Following three consecutive collections of $30 \mu \mathrm{L}$ of effluent from each group, the concentration of effluent drug was determined. Recovery efficiency was calculated based on the concentration of the effluent drug over the standard concentration of drug. For microdialysis sampling, $25 \mu \mathrm{L}$ of exchanged effluent dialysate was collected at different time periods after intraperitoneal injection of carbamazepine or phenytoin. The drug concentration in extracellular cortical fluid was normalized to the recovery efficiency; the drug concentration in cortical extracellular fluid was equal to the drug concentration in dialysate/recovery efficiency.

\section{Measurement of drug concentration by high-performance liquid chromatography}

Concentrations of carbamazepine and phenytoin in serum and dialysate were detected using high-performance liquid chromatography (Model 510, Beijing Syltech Scientific Instrument Co, Ltd, Beijing, People's Republic of China). For measurement of serum drug levels, $200 \mu \mathrm{L}$ of acetonitrile containing $10 \mu \mathrm{g}$ of hexobarbital (as an internal standard) was added to $200 \mu \mathrm{L}$ of serum. This mixture was then agitated for 15 seconds and centrifuged for 10 minutes. The supernatant $(15 \mu \mathrm{L})$ was injected into the chromatography column. For measurement of the dialysate, $1 \mu \mathrm{L}$ of acetonitrile containing hexobarbital was added to $20 \mu \mathrm{L}$ of dialysate. The mixture was left to stand for 10 seconds, and a total of $15 \mu \mathrm{L}$ was then injected into the chromatograph. A $20 \mu \mathrm{L}$ sample was prepared according to the protocol. Chromatographic conditions were as follows: column ODS C18 (200 mm*4 mm, $10 \mu \mathrm{m}$ particle size, Lichrosorb RP); mobile phase, methanol/water (55:45, v:v); flow rate, $1.3 \mathrm{~mL}$ per minute; ultraviolet wavelength of detection, $210 \mathrm{~nm}$; pressure of pump, 1,500 psi; and paper running speed, $0.5 \mathrm{~cm}$ per minute. Minimum detection was $0.4 \mu \mathrm{g} / \mathrm{mL}$ for phenytoin and $0.3 \mu \mathrm{g} / \mathrm{mL}$ for carbamazepine.

\section{Statistical analysis}

All statistical analyses were carried out using SigmaStat (Chicago, IL, USA). Pairwise comparison of P-glycoprotein expression and antiepileptic drug concentration between the groups was done using either the paired Student's $t$-test or one-way analysis of variance as indicated. The data are presented as the mean \pm standard deviation. Differences were considered to be statistically significant at $P<0.05$.

\section{Results}

\section{Selection of phenytoin responders and nonresponders}

Among the 20 kindled rats treated with phenytoin, eight were resistant (nonresponders) and nine showed variable responses to phenytoin (responders). The remaining three rats did not pass the drug selection phase due to variability in the after discharge threshold.

\section{Upregulation of P-glycoprotein in cortex and hippocampus of epileptic rats}

Real-time RT-PCR showed that MDRI (P-glycoprotein mRNA) expression was higher in the cerebral tissue from epileptic rats than in that from normal rats (Figure 1A). Semiquantitative analysis by densitometry illustrated that the amount of $M D R 1$ expression was significantly increased in the hippocampus and cortex of kindled rats (cortex, $P=0.002$; hippocampus, $P=0.013$ [Figure 1B]). However, there was no difference between MDRI levels in the cortex and those in the hippocampus in the control or epilepsy group, indicating that the increase in MDRl levels were characteristic of epilepsy regardless of its distribution in the cerebra. In addition, P-glycoprotein levels were significantly increased 
A

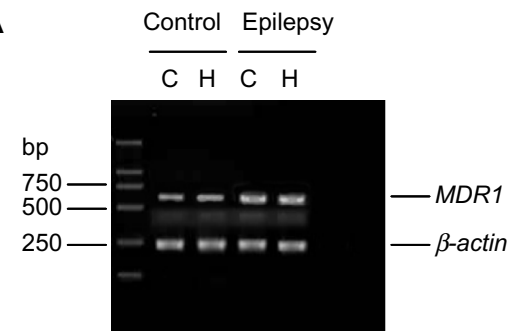

C

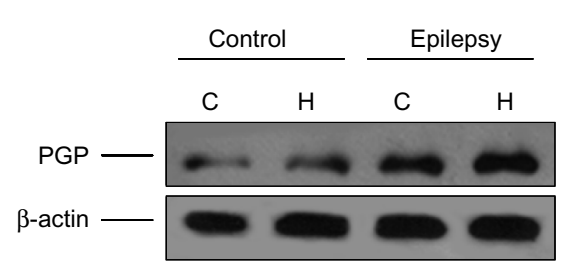

B

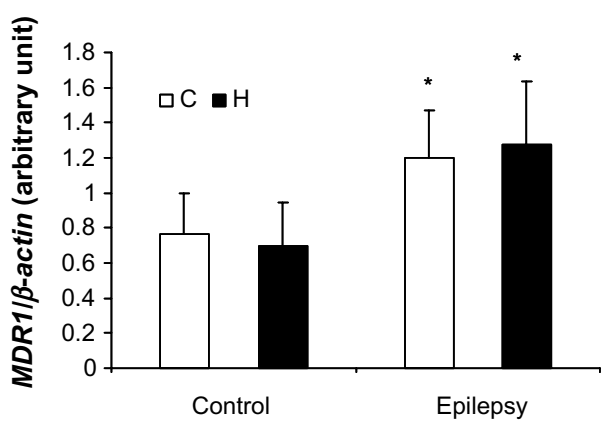

D

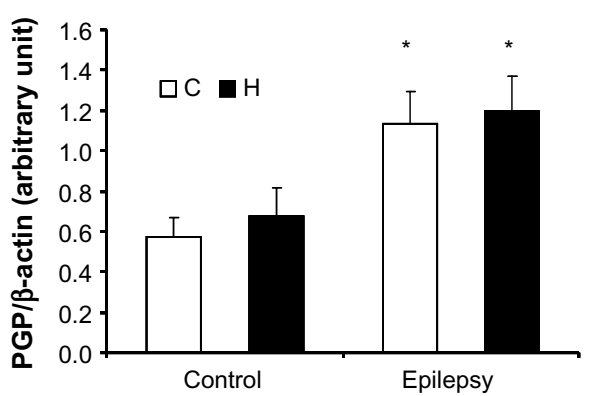

Figure I Expression of P-glycoprotein and its mRNA in cerebral tissue. MDRI was determined using real-time reverse transcription polymerase chain reaction and P-glycoprotein using Western blotting. RNA and total protein was extracted from the hippocampus $(\mathrm{H})$ and cortex $(\mathrm{C})$ of control rats and kindled rats. (A) Products of PCR were run on a I\% w/w agarose gel. $\beta$-actin (lower band, $24 \mathrm{l}$ bp) and MDRI (upper band, $600 \mathrm{bp}$ ) are shown. (B) Semiquantitative analysis using gel after densitometry. The amount of MDRI expression is represented by the ratio of $M D R I$ to $\beta$-actin (mean \pm standard deviation, $\mathrm{n}=8$ ) and normalized. $* \mathrm{P}<0.05$ versus control group. (C) Western blotting of $\mathrm{P}$-glycoprotein, and (D) quantitative analysis. The amount of $\mathrm{P}$-glycoprotein expression was normalized to $\beta$-actin (mean \pm standard deviation, $\mathrm{n}=8$ ). $* \mathrm{P}<0.05$ versus control group.

Abbreviation: PGP, P-glycoprotein.

in cerebral tissue from epileptic rats ( $\mathrm{n}=8$; cortex, $P=0.0003$; hippocampus, $P=0.0001$ ) as shown in Figure $1 \mathrm{C}$ and $\mathrm{D}$.

\section{Inhibition of P-glycoprotein increased antiepileptic drug concentration in extracellular cortical fluid}

Since the serum concentration of drugs is an indicator after the administration of drugs, we first detected the serum phenytoin concentration after intraperitoneal injection. After injection of phenytoin $50 \mathrm{mg} / \mathrm{kg}$, plasma phenytoin levels peaked rapidly and were maintained at 15-45 minutes after administration. The serum phenytoin concentration was $12.29-17.35 \mu \mathrm{g} /$ $\mathrm{mL}$ at $15-180$ minutes post-injection (Figure $2 \mathrm{~A}$ ). Next, we measured concentrations of phenytoin in extracellular cortical fluid after it had passed through the blood-brain barrier. In the control rats, phenytoin rapidly crossed the blood-brain barrier into cerebral tissue, and was detectable in extracellular cortical fluid at 15 minutes after administration. The highest phenytoin concentration in extracellular cortical fluid was $1.24 \pm 0.23 \mu \mathrm{g} /$ $\mathrm{mL}$ at 60 minutes post-injection (Figure 2B), with levels in the range of $0.59-1.24 \mu \mathrm{g} / \mathrm{mL}$ seen over $15-180$ minutes. The ratio of the concentration of phenytoin in extracellular cortical fluid to that in serum was $3 \%-8 \%$. In kindled animals, phenytoin could be detected in extracellular cortical fluid 15 minutes after administration, reaching a peak at 60 minutes, and then gradually decreasing thereafter. The trend of drug concentration in the cortical extracellular fluid of epilepsy was similar to that in normal group. However, compared with controls, phenytoin concentrations in the extracellular cortical fluid of kindled rats was significantly lower than in the control group at each time point over $30-180$ minutes $(P=0.038,0.034$, $0.001,0.011,0.010,0.025$, and 0.007 , respectively, $\mathrm{n}=8)$ as shown in Figure 3A).

As with phenytoin, mean plasma carbamazepine levels peaked at $11.26 \pm 2.12 \mu \mathrm{g} / \mathrm{mL} 15$ minutes after intraperitoneal injection at a $20 \mathrm{mg} / \mathrm{kg}$ dose, and then gradually decreased. Carbamazepine concentrations ranged from 5.94 to $11.26 \mu \mathrm{g} / \mathrm{mL}$ over $15-180$ minutes (Figure $2 \mathrm{C}$ ). In the control group, carbamazepine passed rapidly through the blood-brain barrier into cerebral tissue, as determined by carbamazepine levels in extracellular cortical fluid at 15 minutes after injection of the drug. These peaked at a mean of $1.52 \pm 0.22 \mu \mathrm{g} / \mathrm{mL}$ at 90 minutes post administration (Figure 2D), and ranged from 0.71 to $1.52 \mu \mathrm{g} / \mathrm{mL}$ over 15-180 minutes. The ratio of carbamazepine concentration in extracellular cortical fluid to that in serum ranged from $6 \%$ to $19 \%$. In kindled animals, carbamazepine was detectable in extracellular cortical fluid at 15 minutes post 

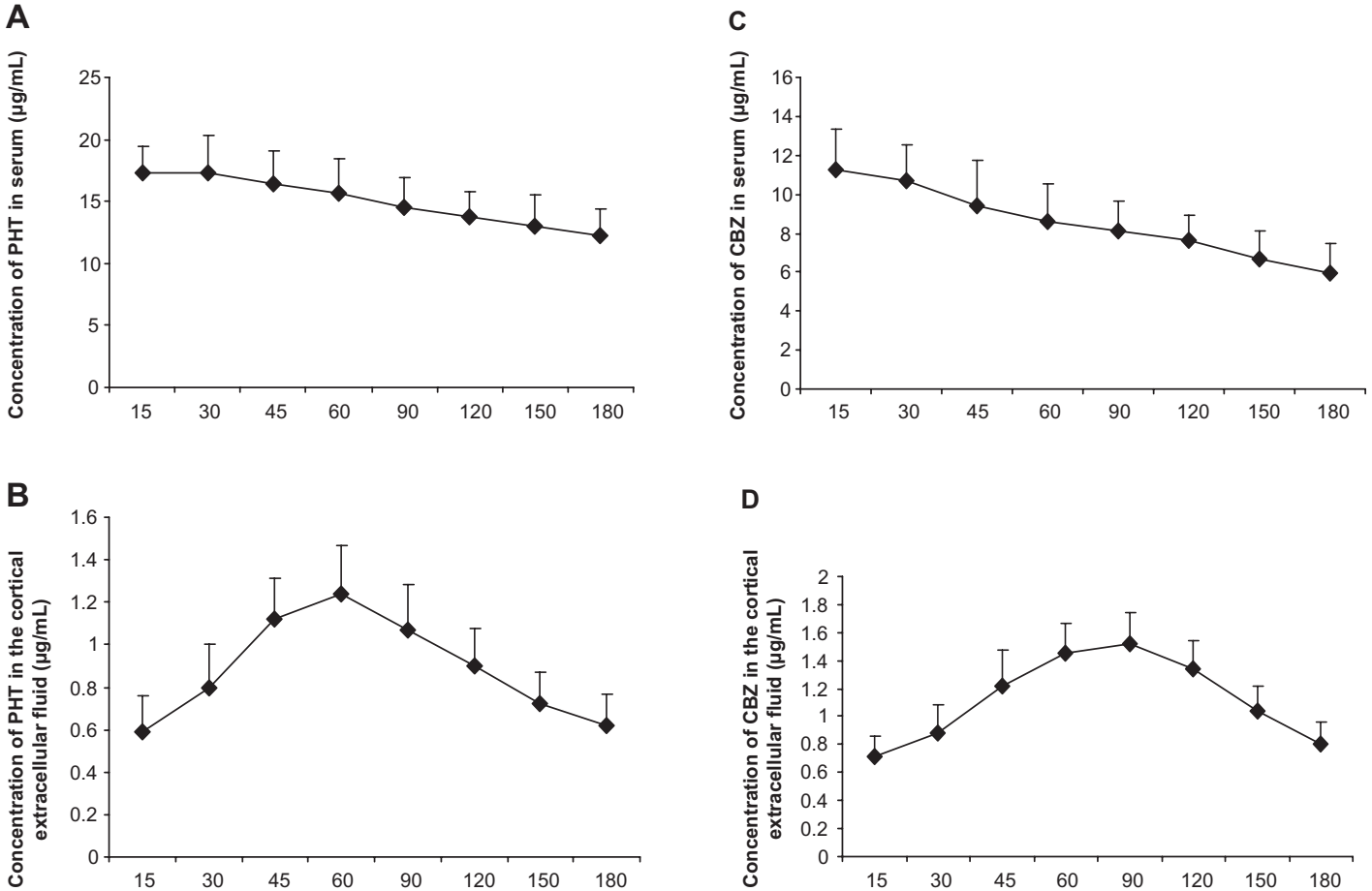

Figure 2 Concentrations of carbamazepine and phenytoin in extracellular cortical fluid and serum.

Notes: Serum concentrations $(\mathbf{A}, \mathbf{C})$ and the cortical extracellular fluid (B,D) of AEDs were detected at the time indicated (post-injection). The highest PHT concentration in serum and the cortical extracellular fluid was at 30 minutes and 60 minutes, respectively, after intraperitoneal injection with $50 \mathrm{mg} / \mathrm{kg} \mathrm{PHT}$ ( $\mathrm{n}=8$ ), and the highest $\mathrm{CBZ}$ concentration in serum and the cortical extracellular fluid was at 15 minutes and 90 minutes, respectively, after intraperitoneal injection with $20 \mathrm{mg} / \mathrm{kg} \mathrm{CBZ}$ ( $\mathrm{n}=8$ ).

Abbreviations: CBZ, carbamazepine; PHT, phenytoin; AED, antiepileptic drug.

administration, reaching a peak at 90 minutes, and then gradually decreased. The fluctuation trend in extracellular cortical fluid was similar in the epilepsy and control groups. However, the carbamazepine level found in extracellular cortical fluid from kindled rats was much lower than that in controls at each time point over 30-150 minutes ( $P=0.024$, $0.014,0.016,0.006,0.028$, and 0.016 , respectively, $\mathrm{n}=8$ ), as shown in Figure 3B.

In addition, verapamil, a P-glycoprotein inhibitor, did not change the concentration trend of phenytoin and carbamazepine, but significantly increased antiepileptic drug concentrations in extracellular cortical fluid from rats with epilepsy at each time point in the phenytoin group (Figure $3 \mathrm{~A}, \mathrm{n}=8, P=0.024,0.003,0.015,0.031,0.028,0.005$, 0.008 , and 0.016 versus epilepsy group, respectively) and at 45,60 , and 120 minutes in the carbamazepine group (Figure $3 \mathrm{~B}, \mathrm{n}=8, P=0.028,0.016$, and 0.036 versus epilepsy group, respectively). The phenytoin concentration in rats with epilepsy after treatment with verapamil was similar to that in control group, indicating that inhibition of P-glycoprotein alters the ability of antiepileptic drugs to penetrate the blood-brain barrier, restoring the accumulation of phenytoin in extracellular cortical fluid.

\section{Discussion}

P-glycoprotein is known as $\mathrm{ABCB} 1$ and belongs to the ATP-binding protein superfamily. ${ }^{15}$ The characteristics of this molecule include two nucleotide-binding domains and three membrane-spanning domains, the function of which appears to involve an ATP-dependent drug pump. Organic anions and pharmaceutics are pumped across the plasma membrane. ${ }^{16,17}$ As a key multidrug transporter in the cerebrum, P-glycoprotein is involved in the functioning of the blood-cerebrospinal and blood-brain barriers, which can remove excess brain metabolites and prevent toxic exogenous substances from entering into the brain tissue and so help to maintain the stability of the brain environment. ${ }^{18-20}$ In normal conditions, expression of P-glycoprotein at both the protein and mRNA levels is low in the hippocampus and cortex, as we observed, suggesting that low P-glycoprotein is sufficient for cerebral homeostasis. ${ }^{21,22}$

However, in pathologic conditions, such as medically intractable epilepsy and glioma, P-glycoprotein expression in capillary endothelial cells and astrocytes is increased. ${ }^{23-25}$ Our research confirms that P-glycoprotein is highly expressed in the cerebrum of rats with medically intractable epilepsy, as detected by real- 


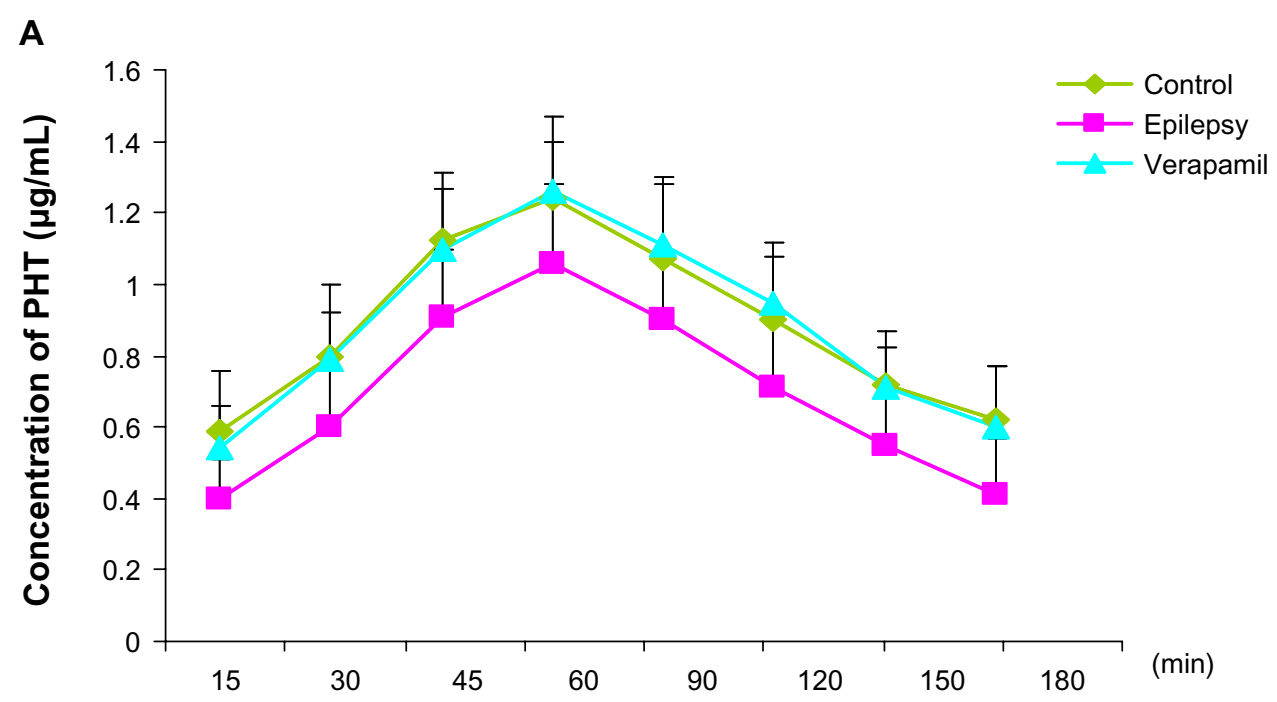

B

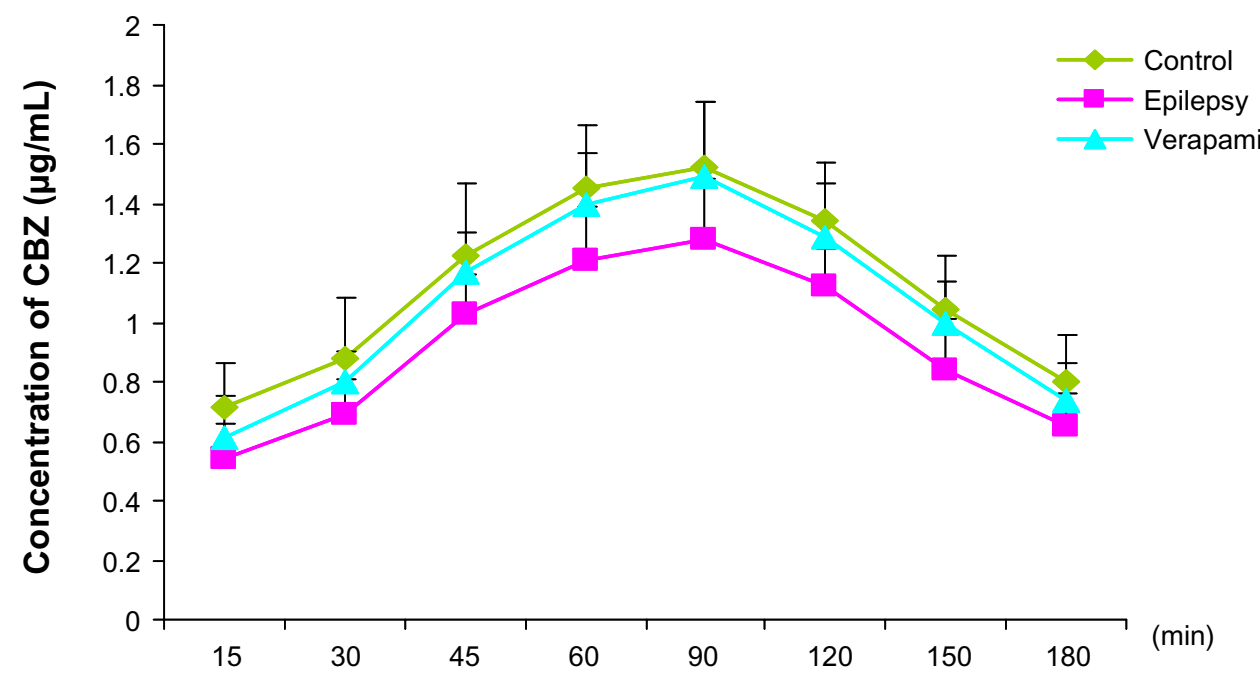

Figure 3 Concentrations of phenytoin (A) and carbamazepine (B) in extracellular cortical fluid as measured by high-performance liquid chromatography.

Notes: Both CBZ and PHT in the cortical extracellular fluid of kindled rats were very lower than those in controls at each time point during 30-180 minutes and 30-150 minutes respectively $(n=8)$. The administration of verapamil significantly restored $P H T$ concentrations at each time point $(\mathbf{A}, n=8, P=0.024,0.003,0.015,0.03 I, 0.028,0.005$, 0.008 and 0.016 versus epilepsy group, respectively), and CBZ concentrations at 45 minutes, 60 minutes and I20 minutes (B, $n=8, P=0.028,0.016$ and 0.036 vs epilepsy group, respectively).

Abbreviations: CBZ, carbamazepine; PHT, phenytoin.

time PCR and Western blotting. Previously, cell lines have been used in vitro and gene knockout rats have been used in vivo; however, we used the rat amygdala kindling model which can effectively mimic uncontrolled seizures in humans.

The findings of our research are in agreement with those of other recent studies showing that high expression of P-glycoprotein may be involved in drug resistance. ${ }^{26-29}$ The present data provide evidence that carbamazepine and phenytoin are substrates for P-glycoprotein at the blood-brain barrier. Thus, overexpression of such efflux transporters in focal epileptogenic cerebral tissue may impair the ability of an antiepileptic drug to pass through the blood-brain barrier. P-glycoprotein can pump antiepileptic drugs out of cerebral cells and/or tissue, decreasing drug concentrations in the extracellular cortical fluid, which could explain why when the first antiepileptic drug prescribed was not effective, switching to a combination of antiepileptic drugs or to another antiepileptic drug still yielded similarly negative results, despite the various pharmacologic mechanisms of antiepileptic drug action.

Verapamil has been considered to be able to antagonize P-glycoprotein function effectively. ${ }^{30-33}$ By inhibiting P-glycoprotein with verapamil, we found that the 
concentration of phenytoin was significantly increased in extracellular cortical fluid from animals with medically intractable epilepsy. Similarly, verapamil markedly increased carbamazepine concentrations in extracellular cortical fluid. However, it remains controversial whether carbamazepine is a substrate for P-glycoprotein. One view is that carbamazepine undergoes extensive metabolism, with the initial oxidative pathways catalyzed by cytochrome (CYP3)A4 and CYP2C $8,{ }^{34}$ and there is overlap between substrates for CYP3A4 and P-glycoprotein. ${ }^{35}$ Therefore, the effect of verapamil on carbamazepine is probably due to inhibition of CYP3A4 and not P-glycoprotein. ${ }^{36}$ However, our investigations confirm that the decreased concentrations of carbamazepine and phenytoin in extracellular cortical fluid are associated with upregulation of P-glycoprotein in the epileptogenic cerebrum. Therefore, further studies are needed to clarify the relationship between P-glycoprotein and carbamazepine in drug resistance.

The mechanism by which P-glycoprotein comes to be highly expressed in cerebral tissue from rats with medically intractable epilepsy is unclear. However, two studies have shown that chemotherapeutic agents and chemical carcinogens, such as barbiturates, cisplatin, and the antibiotic rifampicin, can induce P-glycoprotein and increase its expression. ${ }^{37,38}$ Thus, antiepileptic drugs may induce an increase in P-glycoprotein expression in epileptogenic cerebral tissue. Other studies have shown that selection of antiepileptic drugs is crucial for new patients with epilepsy, especially in childhood. The initial response to antiepileptic drug treatment is highly predictive of the long-term outcome, and appropriate drug selection should avoid drug resisitance. ${ }^{39-41}$ In addition, seizures may upregulate the expression of P-glycoprotein.

In conclusion, our findings demonstrate that $\mathrm{P}$-glycoprotein is upregulated in the epileptic cerebrum. Increased P-glycoprotein levels reduced the ability of antiepileptic drugs to penetrate the blood-brain barrier in rats with medically intractable epilepsy, whereas inhibition of P-glycoprotein improved antiepileptic drug concentrations in extracellular cortical fluid, suggesting that P-glycoprotein is involved in resistance to antiepileptic drugs in medically intractable epilepsy.

\section{Acknowledgments}

This investigation was supported by grants from the Natural Science Foundation of Shanghai (09ZR1405500) and the Shanghai Municipal Health Bureau (2008-08) to YC.

\section{Disclosure}

The authors report no conflicts of interest in this work.

\section{References}

1. Elia M, Amato C, Bottitta M, et al. An atypical patient with Cowden syndrome and PTEN gene mutation presenting with cortical malformation and focal epilepsy. Brain Dev. 2012;34(10): 873-876.

2. Elia M, Falco M, Ferri R, et al. CDKL5 mutations in boys with severe encephalopathy and early-onset intractable epilepsy. Neurology. 2008;23;71(13):997-999.

3. Capovilla G, Beccaria F, Montagnini A, et al. Short-term nonhormonal and nonsteroid treatment in West syndrome. Epilepsia. 2003;44(8): $1085-1088$.

4. Loscher W, Potschka H. Role of drug efflux transporters in the brain for drug disposition and treatment of brain diseases. Prog Neurobiol. 2005;76(1):22-76.

5. Potschka H, Loscher W. Multidrug resistance-associated protein is involved in the regulation of extracellular levels of phenytoin in the brain. Neuroreport. 2001;12(11):2387-2389.

6. Tishler DM, Weinberg KI, Hinton DR, Barbaro N, Annett GM, Raffel C. MDR1 gene expression in brain of patients with medically intractable epilepsy. Epilepsia. 1995;36(1):1-6.

7. Seegers U, Potschka H, Loscher W. Transient increase of P-glycoprotein expression in endothelium and parenchyma of limbic brain regions in the kainate model of temporal lobe epilepsy. Epilepsy Res. 2002;51(3): $257-268$.

8. Morimoto K, Sato H, Sato K, Sato S, Yamada N. BW1003C87, phenytoin and carbamazepine elevate seizure threshold in the rat amygdale-kindling model of epilepsy. Eur J Pharmacol. 1997;339(1):11-15.

9. Hung CC, Chen CC, Lin CJ, Liou HH. Functional evaluation of polymorphisms in the human ABCB1 gene and the impact on clinical responses of antiepileptic drugs. Pharmacogenet Genomics. 2008; 18(5):390-402.

10. Potschka H, Fedrowitz M, Loscher W. P-glycoprotein and multidrug resistance-associated protein are involved in the regulation of exrracellular levels of the major antiepileptic drug carmbamazepine in the brain. Neuroreport. 2001;12(6):3557-3560.

11. Baltes S, Gastens AM, Fedrowitz M, Potschka H, Kaever V, Löscher W. Differences in the transport of the antiepileptic drugs phenytoin, levetiracetam and carbamazepine by human and mouse P-glycoprotein. Neuropharmacology. 2007;52(2):333-346.

12. Morimoto K, Goddard GV. Seizure-triggering mechanism in the kindling model of epilepsy: I. EEG changes during stimulation from the site of stimulation. Jpn J Psychiatry Neurol. 1988;42(3):618-619.

13. Racine RJ. Modification of seizure activity by electrical stimulation. II. Motor seizure. Electroencephalogr Clin Neurophysiol. 1972;32(3):281-294.

14. Löscher W, Rundfeldt C. Kindling as a model of drug-resistant partial epilepsy: selection of phenytoin-resistant and nonresistant rats. J Pharmacol Exp Ther. 1991;258(2):483-489.

15. Sharom FJ. ABC multidrug transporters: structure, function and role in chemoresistance. Pharmacogenomics. 2008;9(1):105-127.

16. Polgar $\mathrm{O}$, Bates SE. ABC transporters in the balance: is there a role in multidrug resistance? Biochem Soc Trans. 2005;33(1):241-245.

17. Choudhuri S, Klaassen CD. Structure, function, expression, genomic organization, and single nucleotide polymorphisms of human $\mathrm{ABCB} 1$ (MDR1), ABCC (MRP), and ABCG2 (BCRP) efflux transporters. Int J Toxicol. 2006;25(4):231-259.

18. Lee G, Dallas S, Hong M, Bendayan R. Drug transporters in the central nervous system: brain barriers and brain parenchyma considerations. Pharmacol Rev. 2001;53(4):569-596. 
19. Shen $\mathrm{S}$, Zhang W. ABC transporters and drug efflux at the blood-brain barrier. Rev Neurosci. 2010;21(1):29-53.

20. Ohtsuki S, Terasaki T. Contribution of carrier-mediated transport systems to the blood-brain barrier as a supporting and protecting interface for the brain; importance for CNS drug discovery and development. Pharm Res. 2007;24(9):1745-1758.

21. Loscher W, Potschka H. Blood-brain barrier active efflux transporters: ATP-binding cassette gene family. NeuroRx. 2005;2(1):86-98.

22. Elsinga PH, Hendrikse NH, Bart J, Vaalburg W, Waarde A. PET studies on P-glycoprotein function in the blood-brain barrier: how it affects uptake and binding of drugs within the CNS. Curr Pharm Des. 2004;10(13):1493-1503.

23. Loscher W, Potschka H. Role of multidrug transporters in pharmacoresistance to antiepileptic drugs. J Pharmacol Exp Ther. 2002;301(1): $7-14$.

24. Sisodiya SM, Lin WR, Harding BN, Squier MV, Thom M. Drug resistance in epilepsy: expression of drug resistance proteins in common cause of refractory epilepsy. Brain. 2002;125(1):22-31.

25. Volk HA, Potschka H, Loscher W. Increased expression of the multidrug transporter P-glycoprotein in limbic brain regions after amygdalakindled seizures in rats. Epilepsy Res. 2004;58(1):67-79.

26. Jeub M, Beck H, Siep E, et al. Effect of phenytoin on sodium and calcium currents in hippocampal CA1 neurons of phenytoin-resistant kindled rats. Neuropharmacology. 2002;42(1):107-116.

27. Potschka H, Loscher W. In vivo evidence for P-glycoprotein-mediated transport of phenytoin at the blood-brain barrier of rats. Epilepsia. 2001;42(10):1231-1240.

28. Lazarowski A, Czornyj L, Lubienieki F, Girardi E, Vazquez S, D'Giano C. ABC transporters during epilepsy and mechanisms underlying multidrug resistance in refractory epilepsy. Epilepsia. 2007;48(5): 140-149.

29. Rizzi M, Caccia S, Guiso, et al. Limbic seizures induce P-glycoprotein in rodent brain: functional implications for pharmacoresistance. J Neurosci. 2002;22(14):5833-5839.
30. Iannetti P, Spalice A, Parisi P. Calcium-channel blocker verapamil administration in prolonged and refractory status epilepticus. Epilepsia. 2005;46(6):967-969.

31. Summers MA, Moore JL, McAuley JW. Use of verapamil as a potential P-glycoprotein inhibitor in a patient with refractory epilepsy. Ann Pharmacother. 2004;38(10):1631-1634.

32. Jambroszyk M, Tipold A, Potschka H. Add-on treatment with verapamil in pharmacoresistant canine epilepsy. Epilepsia. 2011;52(2):284-291.

33. Pirker S, Baumgartner C. Termination of refractory focal status epilepticus by the P-glycoprotein inhibitor verapamil. Eur J Neurol. 2011;18(12):e151.

34. Kerr BM, Thummel KE, Wurden CJ, et al. Human liver carbamazepine metabolism - role of CYP3A4 and CYP2C8 in 10,11-epoxide formation. Biochem Pharmacol. 1994;47(11):1969-1979.

35. Kim RB, Wandel C, Leake B, et al. Interrelationship between substrates and inhibitors of human CYP3A and P-glycoprotein. Pharm Res. 1999;16(3):408-414.

36. Owen A, Pirmohamed M, Tettey JN, et al. Carbamazepine is not a substrate for P-glycoprotein. Br J Clin Pharmacol. 2001;51(4):345-349.

37. Lu Y, Yan Y, Wang XF. Antiepileptic drug-induced multidrug resistance P-glycoprotein overexpression in astrocytes cultured from rat brains. Chin Med J. 2004;117(11):1682-1686.

38. Felix RA, Barrand MA. P-glycoprotein expression in rat brain endothelial cells: evidence for regulation by transient oxidative stress. $J \mathrm{Neu}$ rochem. 2002;80(1):64-72.

39. Coppola G, Auricchio G, Federico R, Carotenuto M, Pascotto A. Lamotrigine versus valproic acid as first-line monotherapy in newly diagnosed typical absence seizures: an open-label, randomized, parallelgroup study. Epilepsia. 2004;45(9):1049-1053.

40. Coppola G, Licciardi F, Sciscio N, Russo F, Carotenuto M, Pascotto A. Lamotrigine as first-line drug in childhood absence epilepsy: a clinical and neurophysiological study. Brain Dev. 2004;26(1):26-29.

41. Löscher W. How to explain multidrug resistance in epilepsy? Epilepsy Curr. 2005;5(3):107-112.
Drug Design, Development and Therapy

\section{Publish your work in this journal}

Drug Design, Development and Therapy is an international, peerreviewed open-access journal that spans the spectrum of drug design and development through to clinical applications. Clinical outcomes, patient safety, and programs for the development and effective, safe, and sustained use of medicines are a feature of the journal, which

\section{Dovepress}

has also been accepted for indexing on PubMed Central. The manuscript management system is completely online and includes a very quick and fair peer-review system, which is all easy to use. Visit http://www.dovepress.com/testimonials.php to read real quotes from published authors. 